\title{
Salicylic Acid Inhibits Indeterminate-Type Nodulation But Not Determinate-Type Nodulation
}

\author{
Paulina C. van Spronsen, ${ }^{1}$ Teun Tak, ${ }^{1}$ Anita M. M. Rood, ${ }^{2}$ Anton A. N. van Brussel, ${ }^{1}$ Jan W. Kijne, ${ }^{1}$ and \\ Kees J. M. Boot ${ }^{1}$ \\ ${ }^{1}$ Institute of Molecular Plant Sciences, Clusius Laboratory, Leiden University, Wassenaarseweg 64, 2333 AL Leiden, The \\ Netherlands. ${ }^{2}$ Leiden Institute of Chemistry, Gorlaeus Laboratory, Leiden University, P.O. Box 9502, 2300 RA Leiden, The \\ Netherlands
}

Submitted 13 June 2002. Accepted 28 September 2002.

\begin{abstract}
LCOs (lipochitin oligosaccharides, Nod factors) produced by the rhizobial symbiote of Vicia sativa subsp. nigra (vetch, an indeterminate-type nodulating plant) are mitogenic when carrying an 18:4 acyl chain but not when carrying an 18:1 acyl chain. This suggests that the 18:4 acyl chain specifically contributes to signaling in indeterminatetype nodulation. In a working hypothesis, we speculated that the 18:4 acyl chain is involved in oxylipin signaling comparable to, for example, signaling by derivatives of the 18:3 fatty acid linolenic acid (the octadecanoid pathway). Because salicylic acid (SA) is known to interfere with oxylipin signaling, we tested whether nodulation of vetch could be affected by addition of $10^{-4} \mathrm{M}$ SA. This concentration completely blocked nodulation of vetch by Rhizobium leguminosarum bv. viciae and inhibited the mitogenic effect of 18:4 LCOs but did not affect LCO-induced root-hair deformation. SA did not act systemically, and only biologically active SA derivatives were capable of inhibiting nodule formation. SA also inhibited $R$. leguminosarum bv. viciae association with vetch roots. In contrast, addition of SA to Lotus japonicus (a determinate-type nodulating plant responding to 18:1 LCOs) did not inhibit nodulation by Mesorhizobium loti. Other indeterminate-type nodulating plants showed the same inhibiting response toward SA, whereas SA did not inhibit the nodulation of other determinate-type nodulating plants. SA may be a useful tool for studying fundamental differences between signal transduction pathways of indeterminate- and determinate-type nodulating plants.
\end{abstract}

Gram-negative soil bacteria belonging to the genera Rhizobium, Bradyrhizobium, Sinorhizobium, Mesorhizobium and Azorhizobium, collectively called rhizobia, can establish symbiotic interactions with plants that belong to the Fabaceae family. During this symbiosis, new root-located organs, the nodules, are formed, in which differentiated bacteria fix nitrogen for the plant. In return, the plant provides carbon to the rhizobia. This symbiotic association is based on an intensive signal exchange between both partners and on a high-level of hostplant specificity. Leguminous plants excrete flavonoids, which interact with one or more bacterial regulatory NodD proteins to induce the expression of nodulation genes, encoding enzymes involved in production and secretion of lipochitin oligosaccharide (LCO) signal molecules. These LCOs are primarily responsible for the formation of a nodule. Plant-produced flavon-

Corresponding author: P. C. van Spronsen; Fax 31-71-5274999; E-mail: spronsen@rulbim.leidenuniv.nl. oids as well as bacterial LCOs contribute to the strict hostplant specificity. The general structure of LCOs is the same for all rhizobia. Normally, LCOs consist of tetra- or pentamers of $\beta$-1, 4-linked N-acetyl-D-glucosamine, with a fatty acid attached to the nitrogen of the nonreducing sugar. Specificity is obtained by various chemical additions to the core structure (Kamst et al. 1998; Stougaard 2000).

Bacteria attach to root hairs and induce root hair deformation as well as root hair curling and the formation of an infection thread, while at the same time, cortical cells inside the root start to divide to form a nodule primordium. The infection thread containing the rhizobia grows towards the nodule primordium, and finally, penetrates the nodule cells (Stougaard 2000). The number of root nodules that are formed is controlled by a process called autoregulation; already induced or existing nodules inhibit formation of new nodules (van Brussel et al. 2002). The most common types of nodules are determinate nodules and indeterminate nodules. For determinate-type nodulation, cell divisions start in the outer root cortex. For indeterminate-type nodulation, cells in the inner root cortex start to divide. Plants that form determinate nodules, such as soybean, bean, and Lotus japonicus, grow mostly at subtropical or tropical locations, whereas indeterminate-type nodulating plants, such as pea, vetch, alfalfa, and clover, are mainly found in more temperate regions. Interestingly, LCOs produced by rhizobia inducing formation of indeterminate nodules carry a polyunsaturated fatty acyl chain, whereas rhizobia that induce formation of determinate nodules produce LCOs carrying a saturated or monounsaturated acyl chain (Kamst et al. 1998). Furthermore, the presence of a polyunsaturated fatty acid has been shown to be essential for induction of nodule formation in indeterminate-type nodulating plants. LCOs produced by the specific symbiote of Vicia sativa subsp. nigra (vetch, an indeterminate-type nodulating plant) are mitogenic when carrying an 18:4 acyl chain (NodRlv-IV/V[18:4,Ac]), but not when carrying an 18:1 acyl chain (NodRlv-IV/V[18:1,Ac]) (Spaink et al 1991). Apparently, the $18: 4$ acyl chain specifically contributes to signaling in the indeterminate type of nodulation.

Polyunsaturated fatty acids are well known as signal molecules in plants and animals. For instance, arachidonic acid, a 20:4 fatty acid present in animal membranes, is converted to the signal molecule prostaglandin through the key enzyme cyclooxygenase (Marnett et al. 1999). In plants, linolenic acid, an 18:3 fatty acid, is converted through the octadecanoid pathway to oxylipin signal molecules such as jasmonic acid (Schaller 2001). Synthesis of both prostaglandin and jasmonic acid can be inhibited by the addition of salicylic acid (SA) (Marnett et al. 1999; Schaller 2001). We asked whether SA 
would affect nodulation of indeterminate-type nodulating plants. If polyunsaturated fatty acyl chains play a crucial role in signaling during indeterminate-type nodulation, addition of SA may interfere with this process and with nodule formation, whereas in determinate-type nodulating plants no effect of SA would be expected. When testing the effect of SA on both types of nodulation, we found that SA indeed completely inhibits indeterminate-type nodulation but not determinate-type nodulation in plants.

\section{RESULTS}

To test the effects of exogenously applied SA on nodulation, Vicia sativa subsp. nigra was selected as an indeterminate-type nodulating plant and Lotus japonicus as a determinate-type nodulating plant. Unless otherwise indicated, $V$. sativa plants were grown for 7 days and L. japonicus plants for 12 days before being examined. All experiments were done on plants grown on liquid medium.

\section{Effect of SA on nodulation}

of Vicia sativa subsp. nigra and Lotus japonicus.

Seven days after inoculation with Rhizobium leguminosarum bv. viciae strains RBL 5523 or 248, roots of Vicia sativa plants have fully developed nodules. These nodules are usually situated at the upper part of the main root (Fig. 1A). However, seven days after inoculation of $V$. sativa roots with $R$. leguminosarum bv. viciae strain RBL 5523 in the presence of $10^{-4} \mathrm{M}$ exogenously applied SA, nodulation was completely inhibited (Fig. 1B). The same complete inhibition was observed after inoculation with $R$. leguminosarum bv. viciae strain 248 . When roots were allowed to grow for 2 or 3 weeks, a few nodules did develop on lateral roots but not on the main root. We tested the effects of several concentrations of SA on bacterial growth in minimal medium (Jensen) and did not observe differences in growth compared with that in controls, even at $10^{-4} \mathrm{M} \mathrm{SA}$ (data not shown).

Roots of Lotus japonicus grown on liquid medium formed nodules readily. Generally, the nodulation process is slower than with $V$. sativa plants. Twelve days after inoculation with Mesorhizobium loti strain TONO, L. japonicus roots have developed primordia and nodules, ranging from very young to
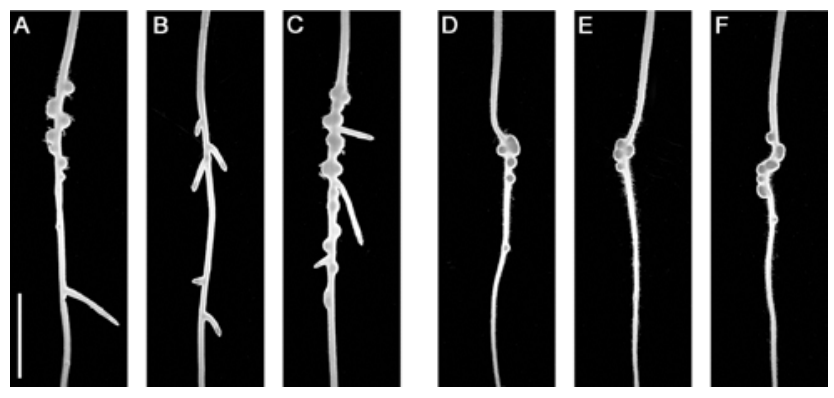

Fig. 1. Light micrographs of root parts in the nodulation zone. Bar $=5$ $\mathrm{mm}$. A through C, Vicia sativa subsp. nigra 7 days after inoculation with Rhizobium leguminosarum bv. viciae strain RBL 5523. A, Control: the nodules are mainly situated at the upper part of the root. B, After addition of $10^{-4} \mathrm{M}$ salicylic acid (SA), nodulation is completely inhibited. C, After addition of $5 \times 10^{-6} \mathrm{M} \mathrm{SA}$, an increased number of nodules have developed, situated mainly at the upper part of the root and distributed over a larger area. D through $\mathbf{F}$, Lotus japonicus 12 days after inoculation with Mesorhizobium loti strain TONO. D, Control: mature nodules are mainly situated at the upper part of the root; less developed primor$\mathrm{dia} /$ nodules distributed over the root are not visible. $\mathbf{E}$, After addition of $10^{-4} \mathrm{M} \mathrm{SA}$, no inhibition of nodulation. $\mathbf{F}$, After addition of $5 \times 10^{-6} \mathrm{M}$ $\mathrm{SA}$, an increased number of mature nodules have developed at the upper part of the root; less developed primordia/nodules distributed over the root are not visible. mature and distributed over the main root (Fig. 1D). Inoculation with two other $M$. loti strains, R7A and E1R, resulted in comparable numbers of nodules and nodule primordia (data not shown). In contrast to the situation with $V$. sativa, exogenous application of $10^{-4} \mathrm{M}$ SA to L. japonicus roots did not inhibit nodulation, and the number of primordia/nodules was comparable to that of untreated control plants (Fig. 1E).

Addition of $10^{-4}$ M SA did not largely affect growth of either V. sativa or L. japonicus. For both plants, an approximately $10 \%$ reduction of root length was observed, but root-hair growth was not affected.

In comparison with $V$. sativa, formation of nodule primordia on L. japonicus roots started about two days later. Therefore, we tested the effect of adding $10^{-4} \mathrm{M} \mathrm{SA}$ at $72 \mathrm{~h}$ after inoculation of $L$. japonicus plants with M. loti strain TONO. The number of nodules formed appeared to be the same as that found on untreated plants or on plants treated with SA at day 0 (data not shown). Apparently, the absence of an inhibiting effect of SA on nodulation of $L$. japonicus is not caused by an immediate metabolization of SA during the first days after its addition.

Addition of SA in concentrations ranging from $10^{-7}$ to $10^{-4}$ $M$ to $V$. sativa roots showed that, at a concentration of $5 \times 10^{-6}$ M SA for R. leguminosarum bv. viciae RBL 5523 and at $5 \times$ $10^{-7}$ M SA for R. leguminosarum bv. viciae 248 , the number of

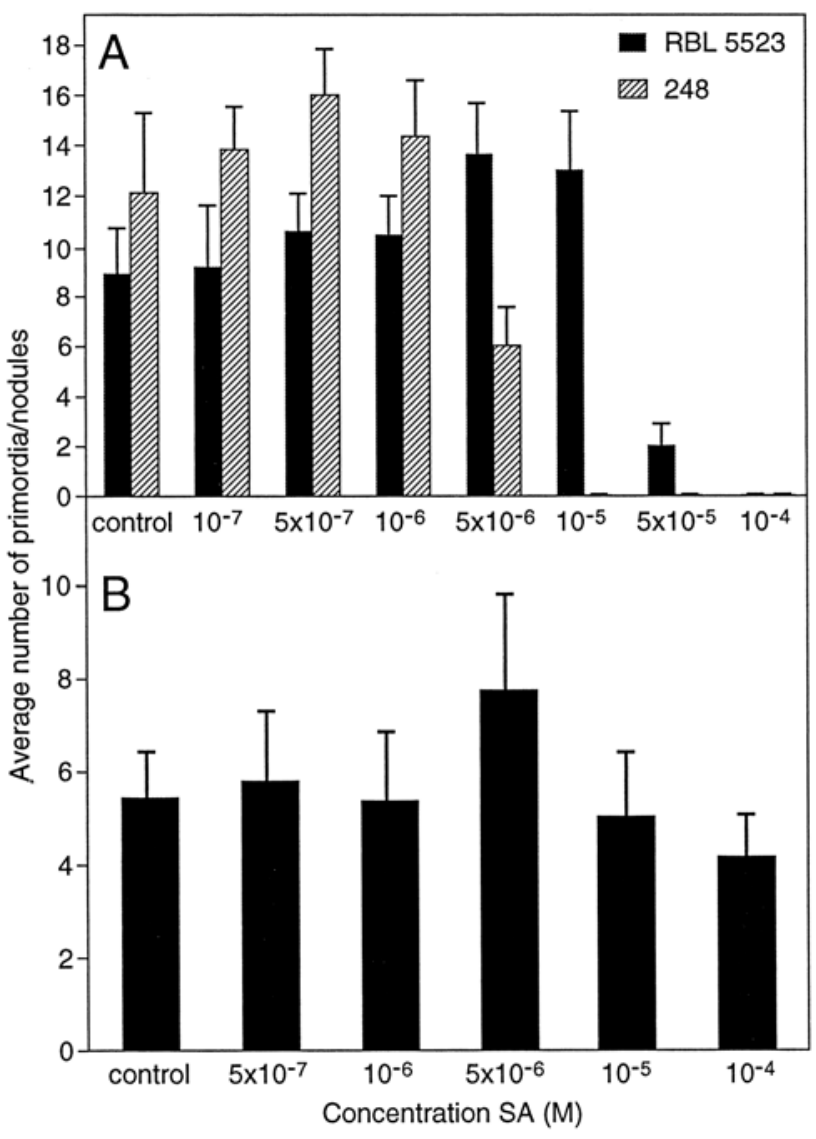

Fig. 2. Effect of salicylic acid (SA) on nodulation of Vicia sativa and Lotus japonicus. A, Nodulation of $V$. sativa roots 7 days after inoculation with Rhizobium leguminosarum bv. viciae. strain RBL 5523 and R. leguminosarum bv. viciae strain 248. R. leguminosarum bv. viciae. RBL 5523 shows significantly increased nodule numbers at a concentration of $5 \times 10^{-6} \mathrm{M} \mathrm{SA}$ and similarly with $R$. leguminosarum bv. viciae. 248 at $5 \times 10^{-7} \mathrm{M} \mathrm{SA}$. With both rhizobia strains, nodulation was completely inhibited at, respectively, $10^{-4} \mathrm{M}$ and $10^{-5} \mathrm{M}$ SA. B, Nodulation of L. japonicus roots 12 days after inoculation with $M$. loti strain TONO. There was a significant increase in number of nodules at a concentration of $5 \times 10^{-6} \mathrm{M} \mathrm{SA}$, and no inhibition of nodulation at $10^{-4} \mathrm{M}$ SA. Bars indicate standard error. 
nodules that developed on the main roots of $V$. sativa is significantly increased ( $t$-test, alpha 0,01 ) after seven days as compared with non-SA-treated plants (Fig. 2A). The nodules were usually situated slightly lower on the roots while the nodulation continued over a longer period, resulting in a larger part of the root being infected (Fig. 1C). Therefore, it seems that, at lower SA concentrations, the autoregulation of $V$. sativa roots is less active, resulting in a prolonged nodulation.

Addition of lower concentrations of SA to L. japonicus roots also gave a significant ( $t$-test, alpha 0,01$)$ stimulation of nodule formation with $M$. loti strain TONO at a concentration of $5 \times$ $10^{-6}$ M SA (Fig. 2B). The primordia and nodules were distributed over the roots in all stages, as in control roots, but there were more nodules present on the higher parts (Fig. 1F) as compared with untreated control roots.

\section{Effect of $10^{-4} \mathrm{M} \mathrm{SA}$ on nodulation of other indeterminate-type and determinate-type nodulating plants.}

In order to test whether the sensitivity of nodulation to $10^{-4} \mathrm{M}$ SA was specific for $V$. sativa, several other indeterminate-type nodulating plants were tested for the effect of $10^{-4} \mathrm{M} \mathrm{SA}$ on nodulation. We used Pisum sativum L. cv. Frisson with strain $R$. leguminosarum bv. viciae 248, Pisum sativum L. cv. Frisson P88 (a supernodulating mutant) with strain $R$. leguminosarum bv. viciae 248, Medicago sativa with Sinorhizobium meliloti strain RCR 2011, and Trifolium repens L. with Rhizobium leguminosarum bv. trifolii strain ANU 843. In each case, a complete inhibitory effect on nodulation was found after addition of $10^{-4} \mathrm{M} \mathrm{SA}$, similar to the situation as described for $V$. sativa. Even on the roots of the supernodulating pea mutant, nodules were completely absent after addition of SA (data not shown).

To test whether the absence of an inhibitory effect of $10^{-4} \mathrm{M}$ SA on nodulation was specific for L. japonicus, several other determinate-type nodulation plants were tested. We used Phaseolus vulgaris cv. Negro Jamapa with $R$. etli strain CE3 and Glycine soja with $S$. fredii strain HNO1. As with $L$. japonicus, the roots of these plants still showed a normal number of nodules after addition of $10^{-4} \mathrm{M} \mathrm{SA}$ (data not shown). Apparently, indeterminate-type nodulation can be completely inhibited by $10^{-4} \mathrm{M} \mathrm{SA}$, whereas determinate-type nodulation is not affected.

\section{Specificity of SA inhibition.}

In order to identify a specific nodulation stage sensitive to SA, a time course of SA addition was performed with $V$. sativa. Addition of $10^{-4} \mathrm{M} \mathrm{SA}$ at 0 up to $72 \mathrm{~h}$ after inoculation with strain $R$. leguminosarum bv. viciae RBL 5523 inhibited nodulation completely. Pretreatment of roots for $24 \mathrm{~h}$ with $10^{-4} \mathrm{M}$ SA before transferring plants to fresh medium without SA and inoculation with strain $R$. leguminosarum bv. viciae RBL 5523 had no significant effect on nodule numbers. Treatment with SA $72 \mathrm{~h}$ after inoculation with strain $R$. leguminosarum bv. viciae RBL 5523 resulted in a 50\% reduction in the number of nodules formed (Fig. 3). From these results, it can be inferred that SA has to be present during the first 3 days of nodulation to yield complete inhibition of nodulation. This period coincides with the period of rhizobial association with the host plant root and with LCO production and activity.

Addition of $10^{-4} \mathrm{M}$ SA to root A of a $V$. sativa split-root system, as recently described by Van Brussel and associates (2002), did not inhibit nodulation of root B, which was inoculated two days later with strain $R$. leguminosarum bv. viciae RBL 5523 (data not shown). Therefore it seems that SA added to root A does not act systemically to affect the nodulation capacity of the spatially separated root B.

Furthermore, we tested the SA specificity of the inhibition of nodulation by adding well-known active and inactive SA ana- logues (Conrath et al. 1995). Figure 4 shows that biologically active SA derivatives, such as acetylSA, 3,5-dichlorobenzoic acid, and 5-chloro-SA, were as active in completely inhibiting indeterminate-type nodulation at $10^{-4} \mathrm{M}$ as was SA. In contrast, well-known biologically inactive analogues such as 3hydroxybenzoic acid and 3,4-dihydroxybenzoic acid were unable to inhibit nodulation of $V$. sativa roots inoculated with $R$. leguminosarum bv. viciae strain RBL 5523 (Fig. 4).

\section{SA affects rhizobial association with $V$. sativa roots.}

To visualize the nodulation process in SA-treated roots of $V$. sativa, we used R. leguminosarum bv. viciae strain RBL 5523 containing plasmid pXLGD4 carrying a fusion of the constitutive hemA promoter to the lacZ reporter gene (Leong et al. 1985; van Workum et al. 1998). $\beta$-galactosidase activity was determined by staining with X-Gal (5-bromo-4-chloro-3-indolyl $\beta$-D-galactoside). Seven days after inoculation, control roots showed stained bacteria situated mainly in the nodules. Occasionally, we observed curled root hairs with either an infection focus or an infection thread. Remarkably, inoculated roots treated with $10^{-4} \mathrm{M} \mathrm{SA}$ did not show any lacZ reporter activity at all (data not shown). This observation suggests that SA at this concentration inhibits association of rhizobia with the host plant root. Microscopic observation of these roots confirmed the absence of firmly attached rhizobia, curled root hairs, and infection threads. However, roots treated with the stimulating concentration of $5 \times 10^{-6} \mathrm{M} \mathrm{SA}$ showed, in addition to stained bacteria in the nodules, an increased number of small activation sites (curled root hairs containing infection threads that have penetrated into a few layers of the cortex) over a large part of the roots (data not shown). These data are in agreement with the observed effects of more primordia/nodule formation and a prolonged nodulation zone seen with stimulating SA concentrations as described above.

In contrast to the situation with $V$. sativa, $\beta$-galactosidase activity in $10^{-4} \mathrm{M}$ SA-treated $L$. japonicus roots inoculated and nodulating with $M$. loti strain TONO, containing the same reporter plasmid pXLGD4, was similar to that in and on control roots (data not shown).

\section{$\mathrm{SA}$ affects $\mathrm{LCO}$ responses in $\boldsymbol{V}$. sativa.}

Absence of a direct rhizobial association with the host plant root does not necessarily preclude induction of formation of nodule primordia by LCOs. Absence of primordium formation in the presence of $10^{-4} \mathrm{M}$ SA suggests that SA also interferes with the

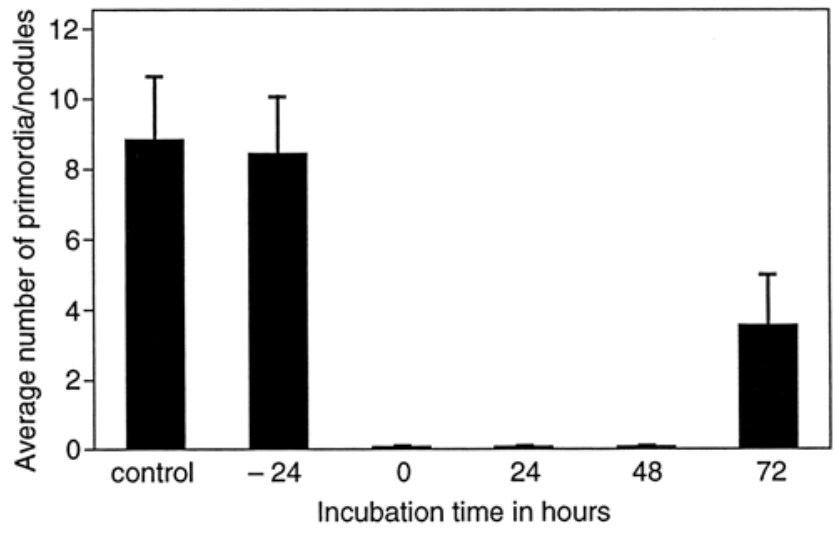

Fig. 3. Time-course incubation with $10^{-4} \mathrm{M}$ salicylic acid (SA) for Vicia sativa inoculated with Rhizobium leguminosarum bv. viciae strain RBL 5523. Pretreatment with SA for $24 \mathrm{~h}$ before transferring to fresh medium without SA and inoculation has no significant effect on nodule numbers. Treatment with SA $72 \mathrm{~h}$ after inoculation decreased nodule numbers by approximately $50 \%$. Addition of SA from 0 to about $72 \mathrm{~h}$ after inoculation inhibited nodulation completely. Bars indicate standard error. 
LCO signaling process. This may occur at several stages, for example, (i) inhibition of the production of nod gene-inducing flavonoids, (ii) inhibition of the production of LCOs, or (iii) inhibition of LCO perception and signal transduction.

$V$. sativa roots were inoculated with $R$. leguminosarum bv. viciae strain RBL 5561 pMP604, containing a flavonoid independent transcription activation (FITA) nodD gene. Addition of $10^{-4}$ M SA strongly (but not completely) inhibited primordium formation (Fig. 5A). Addition of SA to roots of $L$. japonicus that were inoculated with $M$. loti strain E1R pMP604 containing the same FITA nodD gene did not show much difference in primordia/nodule numbers as compared with plants treated with a $M$. loti strain TONO. SA at $10^{-4} \mathrm{M}$ did not inhibit nodulation (data not shown). These results indicate that the inhibiting effect of SA on indeterminate-type nodulation is apparently downstream of flavonoid production.

In order to test whether SA affects LCO signaling during indeterminate-type nodulation, we used purified mitogenic Nod factors to induce nodule primordia formation in vetch. Formation of primordia, which developed on the roots of $V$. sativa after incubation with $N o d R l v-\mathrm{IV} / \mathrm{V}[18: 4, \mathrm{Ac}] \mathrm{LCOs}$ at a concentration of $10^{-7} \mathrm{M}$, was increasingly inhibited by increasing amounts of SA. At $10^{-4} \mathrm{M} \mathrm{SA}$, inhibition was approximately $85 \%$ compared with untreated roots. Addition of lower concentrations of LCOs at $10^{-8} \mathrm{M}$ or $10^{-9} \mathrm{M}$ yielded similar results, with a complete inhibition of primordia formation already at $10^{-5}$ M SA (Fig. 5B).

These data indicate that the amount of LCOs is an important factor for complete inhibition of nodulation by SA. If the concentration of LCOs is relatively high, such as with the constitutively LCO-producing FITA nodD strain or after addition of purified LCOs at a concentration of $10^{-7} \mathrm{M}$, inhibition is not complete. However, at lower concentrations of Nod factor, SA can fully inhibit formation of nodule primordia in $V$. sativa roots.

$V$. sativa root hairs respond with root hair deformations to both mitogenic and nonmitogenic LCOs. To test whether SA interferes with recognition of LCOs by vetch root hairs, we studied the effect of the addition of $10^{-4} \mathrm{M} \mathrm{SA}$ on root hair deformation in the presence of LCOs. $V$. sativa roots treated with $10^{-7} \mathrm{M}$ mitogenic NodRlv-IV/V[18:4,Ac] or nonmitogenic NodRlv-IV/V[18:1,Ac] LCOs showed strong root hair deformations, both in the presence of or in the absence of SA (data

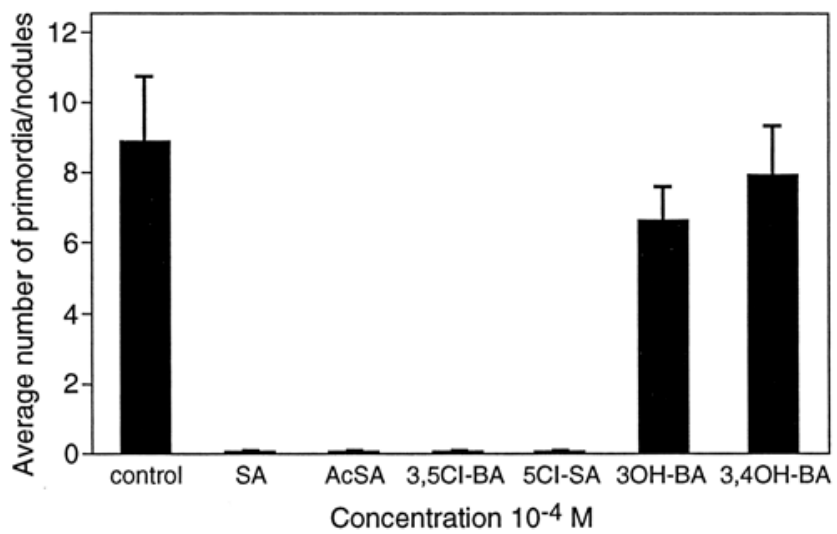

Fig. 4. Nodulation of Vicia sativa roots 7 days after inoculation with Rhizobium leguminosarum bv. viciae strain RBL 5523 and treatment with several biologically active and inactive salicylic acid (SA) analogues at a concentration of $10^{-4} \mathrm{M}$. With the biologically active SA analogues acetylSA (AcSA), 3,5-dichlorobenzoic acid (3,5Cl-BA), and 5chloro-SA (5Cl-SA), the nodulation is completely inhibited. With the biologically inactive analogues 3-hydroxybenzoic acid (3OH-BA) and 3,4 dihydroxybenzoic acid (3,4OH-BA), there is normal nodulation. Bars indicate standard error. not shown). From this observation, we conclude that SA does not affect the recognition of LCOs by vetch root hairs.

Taken together, these results locate one or more of the LCOsignaling stages in vetch that are inhibited by SA downstream from root hair recognition and upstream from induction of primordium formation, provided that these processes are epistatic.

\section{Effect of SA on nodulation of $V$. sativa}

by mutant $R$. leguminosarum bv. viciae strains.

In our working hypothesis, we speculated that the LCO 18:4 acyl chain is involved in oxylipin signaling, leading to induction of nodule primordium formation. The 18:4 fatty acid may function as a precursor for the biosynthesis of an essential oxylipin signal molecule. We studied the effect of SA on the nodulation behavior of certain mutant $R$. leguminosarum bv. viciae strains: (i) $R$. leguminosarum bv. viciae strain RBL 5602 , a nodE mutant that produces only nonmitogenic NodRlvIV/V [18:1,Ac] LCOs, (ii) R. leguminosarum bv. viciae strain RBL 5797, a nodO mutant lacking a secreted putative cationselective channel-forming protein, and (iii) R. leguminosarum bv. viciae strain A91, a nodE nodO double mutant.

$R$. leguminosarum bv. viciae strain RBL 5602, although lacking the mitogenic NodRlv-IV/V [18:4,Ac] LCOs, yielded

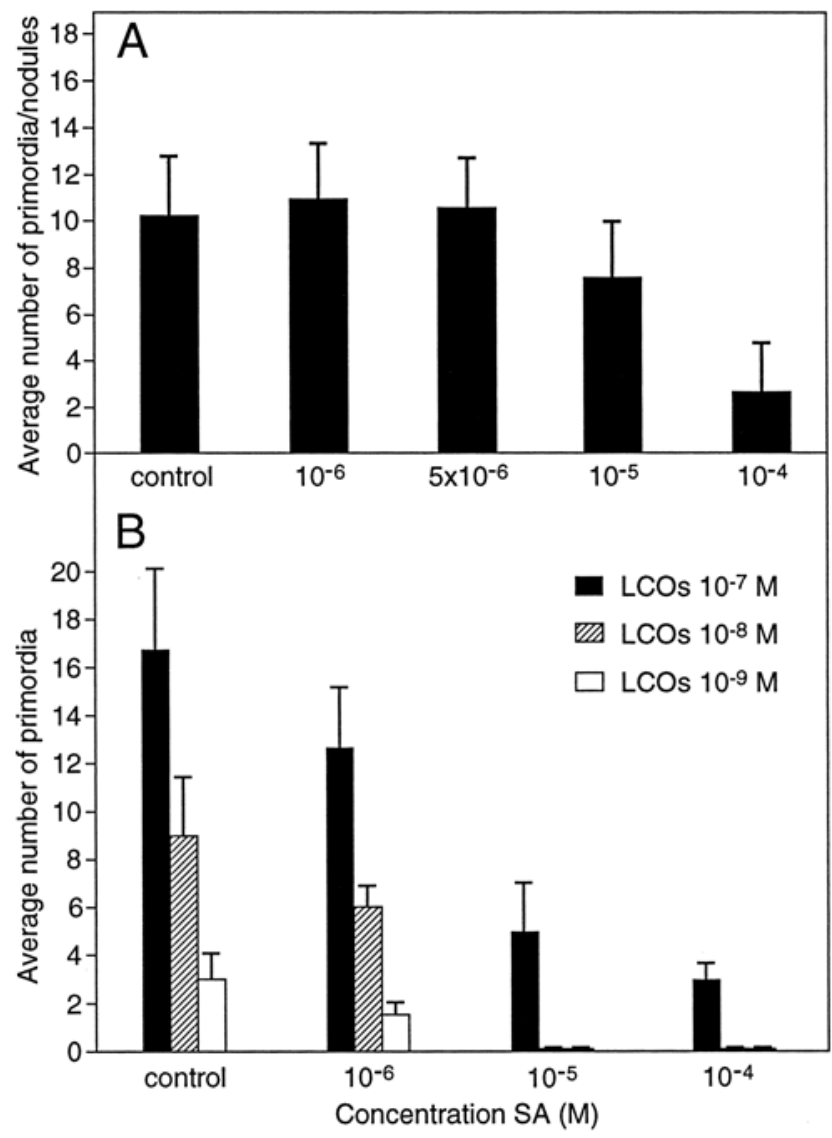

Fig. 5. Effect of salicylic acid (SA) on Vicia sativa after inoculation with a flavonoid independent transcription activation (FITA) nodD gene strain and lipochitin oligosaccharides (LCOs). A, Nodulation of $V$. sativa roots 7 days after inoculation with Rhizobium leguminosarum bv. viciae. strain RBL 5561 pMP 604 containing a FITA nodD gene. Addition of $10^{-4} \mathrm{M}$ $\mathrm{SA}$ resulted in a strong reduction, but not a complete inhibition of the nodulation. B, Formation of primordia in $V$. sativa roots 7 days after inoculation with mitogenic LCOs (NodRlv-IV/V [18:4,Ac]) at $10^{-7}, 10^{-8}$, and $10^{-9} \mathrm{M}$. Increasing concentrations of SA resulted in a gradual inhibition of the formation of primordia. At lower LCO concentrations, primordia formation was already completely inhibited at $10^{-5} \mathrm{M}$ SA. Bars indicate standard error. 
normal nodule formation on $V$. sativa roots. Addition of SA at $10^{-4} \mathrm{M}$ completely inhibited nodulation (Fig. 6). Inoculation with $R$. leguminosarum bv. viciae strain RBL 5797, a nodO mutant, also yielded normal nodulation. Again, SA at $10^{-4} \mathrm{M}$ accomplished a complete inhibition of primordia/nodule formation (Fig. 6). Inoculation with $R$. leguminosarum bv. viciae strain A91, a nodE nodO double mutant that forms multiple aberrant infections but also some nodules on $V$. hirsuta (Walker and Downie 2000), did not result in induction of nodule formation on $V$. sativa subsp. nigra. Control roots developed multiple aberrant infections like those described by Walker and Downie (2000), as seen after X-Gal staining of the A91 lacZ-containing strain. Increasing amounts of SA inhibited formation of these infection sites, while addition of $10^{-4} \mathrm{M}$ SA gave complete inhibition of infection, as with $R$. leguminosarum bv. viciae strain RBL 5523-inoculated plants.

These data confirm earlier observations that mitogenic NodRlv-IV/V [18:4,Ac] LCOs and the nodO protein are mutually redundant for nodulation of vetch. When one of the two is missing, such as in the nod $\mathrm{E}$ or nod $\mathrm{O}$ single mutants, nodulation occurs. Only nodulation of the nod $\mathrm{E} \operatorname{nod} \mathrm{O}$ double mutant is impaired. SA at $10^{-4} \mathrm{M}$ not only inhibits nodule formation with the single mutants, but also the aberrant infections by the double mutant. This can be explained by the inhibiting effect of SA on rhizobial association with the vetch root surface. The nodulation data with the $R$. leguminosarum bv. viciae mutants also indicate that SA does not necessarily inhibit biosynthesis of an 18:4-derived oxylipin signal to inhibit nodulation. Also 18:1 LCO/NodO signaling is affected by SA.

\section{Measurement of levels of free $\mathrm{SA}$ in $\boldsymbol{V}$. sativa roots.}

Recently, it was found that a $R$. leguminosarum bv. viciae nod C mutant strain that is unable to produce any LCOs induced SA accumulation in pea roots, whereas a $R$. leguminosarum bv. viciae 248 wild-type strain did not induce an increase in the level of endogenous SA (Blilou et al. 1999). Similar results were observed during a $R$. meliloti-alfalfa symbiosis (Martínez-Abarca et al. 1998). The authors suggested that rhizobia that could not produce LCOs could elicit a SA-related defense response in legume plants. Therefore, we decided to analyze the accumulation of SA in $V$. sativa roots, after inoculation with various rhizobial strains and purified LCOs. We did not observe an increase in the level of endogenous SA after inoculation of $V$. sativa roots for 24,48 , or 72 h with $R$. leguminosarum bv. viciae strains RBL 5523, RBL 5045 (a Sym-plasmid-cured strain without nod genes), or RBL 5562, a nodA mutant strain. Also, the roots did not show an increase in the free SA level after treatment with purified LCOs (Fig. 7). Furthermore, addition of nonhost rhizobia did not yield an increase in the SA level (data not shown). From these results, we conclude that, in contrast to the situation in pea roots, in $V$. sativa roots, no SA-related defense response is elicited by rhizobia unable to produce LCOs. Addition of exogenous $10^{-6} \mathrm{M} \mathrm{SA}$ to the plant growth medium resulted in an amount of SA in the roots after $24 \mathrm{~h}$ that was 10 times larger than that found in Rhizobium-treated roots (Fig. 7). Addition of $10^{-4} \mathrm{M} \mathrm{SA}$ to the growth medium gave a 1,000-fold increase in the level of free SA (data not shown). Therefore, we conclude that the approximately 10fold increase in the level of free SA that was found in pea roots after inoculation with an LCO-deficient $R$. leguminosarum bv. viciae strain is not sufficient to inhibit the indeterminate-type nodulation of pea.

\section{DISCUSSION}

This study shows that SA affects indeterminate-type nodulation but not determinate-type nodulation. The main differences between these two types of nodulation are (i) the position at which the first root cell divisions take place and (ii) the differentiation of the mature nodule. Formation of indeterminate nodules starts in the inner cortex, and the mature nodule contains a persistent apical meristem that gives rise to several differentiated nodule regions. Indeterminate nodules export nitrogen mainly in the form of asparagine and glutamine. Most determinate nodules are formed in the outer root cortex, and nodule growth occurs through cell expansion rather than through cell division. Although L. japonicus seems to be an intermediate, in that nodule primordia originate from the middle root cortex, it eventually forms a determinate nodule. Determinate nodules export fixed nitrogen in the form of ureides. Differences between indeterminate- and determinatetype nodulation may result from fundamental differences in root physiology between the plant genera concerned.

Apart from these differences, the nodulation process of $V$. sativa and L. japonicus consists of the same stages, with root infection occurring after rhizobial attachment to root hairs, followed by formation of an infection thread and the formation of preinfection thread structures in the root cortex, as well as by cell divisions and nodule formation (Van Spronsen et al. 2001). Therefore, it is interesting to note that SA can completely block indeterminate-type nodulation in all plant species tested, including a supernodulating pea mutant, whereas for all determinate nodule-forming plants tested, addition of SA did not affect nodule number. Most likely, SA specifically affects the physiological state of indeterminately nodulating plants. The inhibitory effect of SA did not appear to result from obvious differential effects on root growth, root hair growth, or root hair formation. Still, a detailed physiological study of the effect of SA on nonnodulated roots from, for example, $V$. sativa and pea versus $L$. japonicus and bean, grown under various conditions, may reveal a difference in response that is pertinent to nodulation.

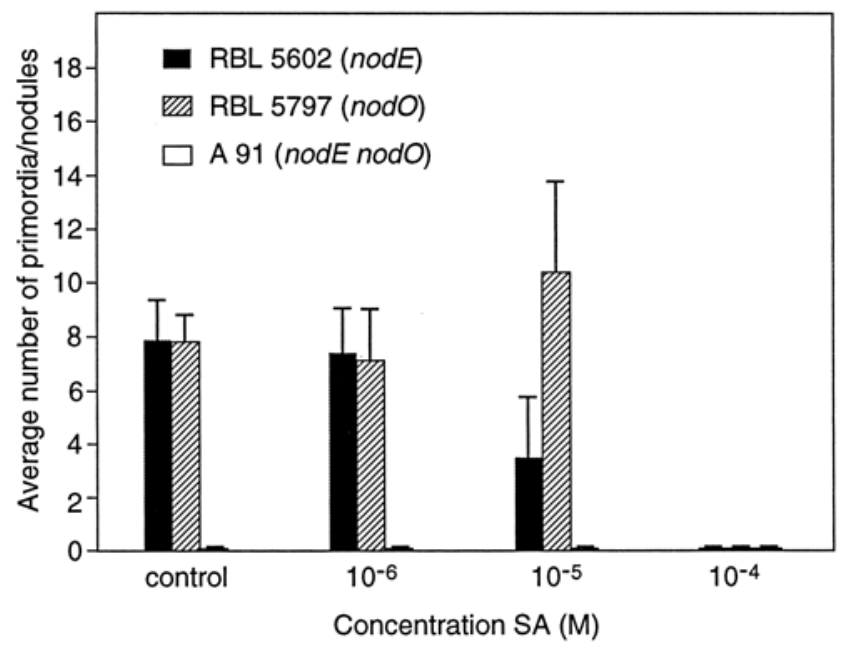

Fig. 6. Nodulation of Vicia sativa roots 7 days after inoculation with various mutant Rhizobium leguminosarum bv. viciae. strains and treatment with a series of salicylic acid (SA) concentrations. Inoculation with $R$. leguminosarum bv. viciae. strain RBL 5602, a nodE only mutant producing nonmitogenic NodRlv-IV/V [18:1,Ac] gave normal nodulation and a complete inhibition at $10^{-4} \mathrm{M}$ SA. Inoculation with R. leguminosarum bv. viciae. strain RBL 5797, a nodO mutant lacking a putative cation-selective channel-forming protein, gave normal nodulation and a complete inhibition at $10^{-4} \mathrm{M}$ SA. No nodules were found after inoculation with $R$. leguminosarum bv. viciae. strain A 91, a nodE nodO double mutant, only aberrant infection sites. Increasing concentrations of SA increasingly inhibited these aberrant infection sites with a complete inhibition at $10^{-4} \mathrm{M} \mathrm{SA}$. Bars indicate standard error. 
From the results obtained with lacZ-containing rhizobial strains, it was apparent that SA inhibits nodulation in indeterminate nodule-forming plants already at the level of association of rhizobia to the root surface. A detailed study of rhizobial attachment in the presence of SA may reveal a specific attachment factor that is negatively influenced. At present, we cannot exclude the possibility that SA influences one or more bacterial attachment factors. If so, these factors apparently are redundant in determinate nodulation. We found that $R$. leguminosarum bv. viciae strain 248 was 10 times more sensitive to SA inhibition of nodulation then $R$. leguminosarum bv. viciae strain RBL 5523. The fact that $R$. leguminosarum bv. viciae strain 248 produces lesser amounts of LCOs then $R$. leguminosarum bv. viciae strain RBL 5523 (H. P. Spaink, personal communication) might be an explanation. Also, LCO signaling in indeterminate nodulation appeared to be inhibited by SA. In a preliminary experiment, we tested whether there were major differences between the amounts of LCOs produced by a determinate nodule-forming rhizobial strain or by an indeterminate nodule-forming strain, but we did not observe significant differences (K. J. M. Boot, unpublished data). Recognition of LCOs by root hairs is not inhibited by SA, judging from the induction of root hair deformation both in the absence of or the presence of $10^{-4} \mathrm{M} \mathrm{SA}$, after the addition of purified LCOs to $V$. sativa roots. However, mitogenic LCOs from $R$. leguminosarum bv. viciae do not induce inner cortical cell divisions in the presence of SA. It has been hypothesized that LCOs are recognized by two types of receptors, a "non-specific" receptor, involved in induction of root hair deformations, and a "specific" receptor, involved in root hair infection and induction of cortical cell divisions (Ardourel et al. 1994). If so, SA negatively affects the specific signal transduction pathway. It remains to be determined whether this inhibitory effect is upstream or downstream of induction of the expression of ENOD40, the early nodulin gene that has been hypothesized to play an essential role in the prelude to nodule primordium formation (Röhrig et al. 2002). The fact that mutant rhizobia such as $n o d \mathrm{E}$ can still induce formation of nodules (in the presence of $\operatorname{nod} \mathrm{O}$ ) rules out a possible role for the polyunsaturated fatty acid chain as a precursor for an as yet unidentified essential signal molecule. Still, the polyunsaturated fatty acyl chain can act as a signal molecule that contributes to a signal transduction pathway, leading to induction of cell division in indeterminate nodulation.

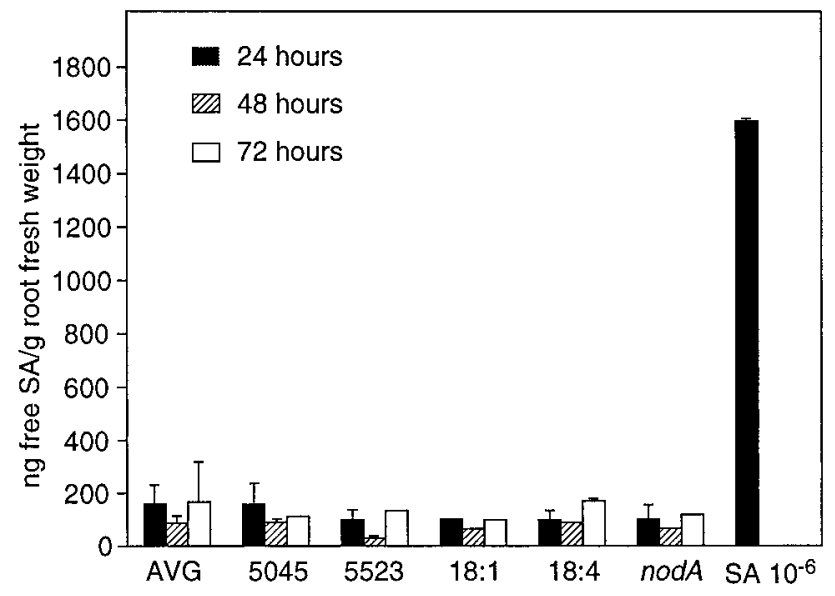

Fig. 7. Measurement of free salicylic acid (SA) levels in Vicia sativa roots at 24,48 , and $72 \mathrm{~h}$ after inoculation with various $R$. leguminosarum bv. viciae. strains and purified lipochitin oligosaccharides (LCOs). No increase in endogenous SA level was found after addition of either bacteria or LCOs. Addition of exogenous $10^{-6}$ M SA resulted in a 10-fold increase in SA level after $24 \mathrm{~h}$. Bars indicate standard error.
At this stage of research, we cannot pinpoint a specific activity of SA responsible for its inhibitory effect. SA has various effects in plants and in plant-microbe interactions. SA induces expression of genes encoding antimicrobial extracellular proteins (Bol et al. 1996). SA binds specifically to iron-containing enzymes, such as catalase, lipoxidase, and peroxidase with an apparent $\mathrm{K}_{\mathrm{d}}$ of around $15 \mu \mathrm{M}$, with production of reactive oxygen species (ROS) and changes in the redox state of plant cells as a result (Rüffer et al. 1995). SA inhibits production of the stress hormone jasmonic acid (Schaller 2001). In addition, SA is a hydrophobic compound that may accumulate in plant membranes, changing membrane fluidity, and as a result, cell physiology (Levitan and Barker 1972). However, this last possibility seems unlikely since acetylsalicylic acid, which was shown to have a 10 times lesser relative ability to alter membrane permeability compared with SA, was as active in inhibiting nodule formation on vetch as was SA.

One target for SA action could be the Rhizobium-induced peroxidase Rip1. Expression of ripl in root tips of Medicago truncatula was found to be correlated with the competence of this zone for symbiotic association. Interestingly, a $M$. truncatula mutant that exhibited a 10 -fold increase in the number of nodules, displayed a correspondingly large increase in ripl transcript levels, while in nonnodulating $M$. truncatula mutant dmil-1, ripl expression by $S$. meliloti was impaired. Furthermore, it was shown that expression levels of ripl were absent in roots that had formed nodules (3 to 7 days postinoculation) (Peng et al. 1996; Ramu et al. 2002). This expression pattern of ripl coincides very well with the inhibitory effect of SA only during the first three days after inoculation that we observed. Addition of $10^{-4} \mathrm{M}$ SA to plants could shift the balance from a symbiotic toward a more defense-like response. There are indications that, also during the initial phase of rhizobia-plant interactions, an oxidative burst is initiated, as was found for pathogen-plant interactions (Bueno et al. 2001; Ramu et al. 2002; Santos et al. 2001). Initial ROS production seems to be a consequence of specific LCO perception, and removal of these ROS could be important for further nodulation to occur. Inhibition of Rip1 or other peroxidases and catalase activity, or both, by SA could result in a stronger and more prolonged oxidative burst and lead to the production of a larger number of ROS. At lower concentrations of SA, an increase in ROS production may stimulate nodulation. However, super optimal $\mathrm{H}_{2} \mathrm{O}_{2}$ production could result in cross-linking of host cell-wall proteins, thereby impairing rhizobial attachment or creating a barrier for rhizobial infection. In addition, ROS could also interfere with 18:4 LCO-signaling that leads to cell division or LCO-induced downregulation of the oxidative burst, as was recently observed in alfalfa cell cultures. Alfalfa host-specific S. meliloti LCOs induced a substantial generation of ROS in tobacco cell cultures but not in alfalfa cells (Baier et al. 1999). It would be interesting to test whether LCOs are able to downregulate the oxidative burst in the presence of various concentrations SA. Our working hypothesis implies that an oxidative burst should not play a major role during determinate-type nodulation. To our knowledge, there are no reports about the production of ROS in determinately nodulating plants at early stages of nodulation.

Our results concerning determination of the level of free $\mathrm{SA}$ in $V$. sativa roots after inoculation of the roots with various host and nonhost rhizobial strains do not support the hypothesis that SA is involved in preventing nodulation of alfalfa and pea in vivo as suggested by Martínez-Abarca and associates (1998) and Blilou and associates (1999). Although a SA concentration of $10^{-6} \mathrm{M}$ has been shown to be biologically relevant for resistance of tobacco leaves to Tobacco mosaic virus (TMV) (Malamy et al 1990, Verberne et al 
2000), it is unknown whether this concentration renders plant roots resistant to root pathogens. Anyway, this concentration is not large enough to cause resistance to rhizobia. Taken together, we consider SA a useful tool to identify differences in rhizobial association mechanisms and signal transduction pathways existing between determinately and indeterminately nodulating plants.

\section{MATERIALS AND METHODS}

\section{Bacterial strains.}

Table 1 shows details about the bacterial strains and their references that were used in this study.

\section{Plant assays.}

Vicia sativa. Seeds of Vicia sativa subsp. nigra were surfacesterilized for $40 \mathrm{~min}$ in concentrated sulfuric acid, were extensively washed, were incubated for $10 \mathrm{~min}$ in commercial bleach, were washed two times in sterile water, and were incubated overnight in $1 \%$ bleach. After three consecutive washings with sterile water, the seeds were spread on $1.8 \%$ agarcontaining Jensen medium (Vincent 1970) in petri dishes and were incubated at $4^{\circ} \mathrm{C}$ for at least 7 days in the dark, upside down. Then, the seeds were allowed to germinate at $28^{\circ} \mathrm{C}$ overnight in the dark. Seeds with roots of about $1.5 \mathrm{~cm}$ long were selected and were transferred to dark pots (with roots shielded from light), with six seeds per pot. These contained liquid Jensen medium with $10 \mathrm{mM}$ MES buffer (ICN Biomedicals, Aurora, OH, U.S.A.), pH 5.6, and the ethylene-biosynthesis inhibitor aminoethoxyvinylglycine (AVG) (Sigma, Bornhem, Belgium) (0.1 mg per 1). The plants were inoculated with bacteria, were grown in yeast manitol broth (YMB) medium (Hooykaas et al. 1977) for 3 days at $28^{\circ} \mathrm{C}$, optical density at $660 \mathrm{~nm}\left(\mathrm{OD}_{660}\right)=10^{-4}$, were diluted 1:1,000 to 1,000 bacteria per $\mathrm{ml}$, and if appropriate, SA in various concentrations or SA analogues (Sigma) were added from stock solutions in water. Then the plants were transferred to a growth chamber at $20^{\circ} \mathrm{C}$ with a $16 \mathrm{~h}$ light and $8 \mathrm{~h}$ dark regime and $70 \%$ relative humidity, and were allowed to grow for 7 days. Mitogenic LCOs NodRlv-IV/V [18:4,Ac] from Rhizobium leguminosarum bv. viciae strain RBL 5601 were purified as described by Spaink and associates (1991). Experiments were done with 12 plants and were repeated at least three times. The possible effect of SA on bacterial growth was measured by taking 100- $\mu$ l samples out of the Jensen medium in which the plants and bacteria had grown for 7 days in the presence of or the absence of SA. Samples were diluted to $10^{-3}$ and $10^{-4}$, and $50 \mu \mathrm{l}$ of these suspensions was put on a plate with tryptone yeast (TY) medium (Priem and Wijffelman 1984) and was grown for 4 days at $28^{\circ} \mathrm{C}$. Bacterial colonies were then counted.

Lotus japonicus. Seeds of Lotus japonicus were sterilized and were allowed to germinate as described previously (van Spronsen et al. 2001). Seedlings with roots about $2.5 \mathrm{~cm}$ long were selected. Plants were grown in liquid medium similar to that described above for $V$. sativa, with the exception of the amount of AVG, which was added in a concentration of 0.025 $\mathrm{mg}$ per 1 . The plants were inoculated with bacteria grown for two days in YMB medium at an $\mathrm{OD}_{660}=10^{-1}$, diluted 1:1,000 to $10^{5}$ bacteria per $\mathrm{ml}$, and if appropriate, SA was added. The plants were transferred to the same growth chamber used for $V$. sativa and were allowed to grow for 12 days. Experiments were done with 12 plants and repeated at least three times.

\section{Other plants.}

Several plants of the indeterminate-nodule-type (Pisum sativum, Trifolium repens, and Medicago sativa) and determinatenodule-type (Glycine soja and Phaseolus vulgaris) were tested for nodulation inhibition by $10^{-4} \mathrm{M} \mathrm{SA}$.

Seeds of $P$. sativum L. cv Frisson and $P$. sativum L. cv Frisson P88, a supernodulating mutant (Sagan and Gresshoff 1996), were surface-sterilized in concentrated sulfuric acid for $10 \mathrm{~min}$, were washed seven times in sterile water, were treated for $15 \mathrm{~min}$ with commercial bleach, were washed three times in sterile water, and were left overnight in $1 \%$ bleach. After thorough washings, the seeds were allowed to germinate for two days between moisturized filter paper at $28^{\circ} \mathrm{C}$. Seedlings with roots from 3 to $6 \mathrm{~cm}$ long were selected and transferred to dark pots containing liquid Jensen medium with $0.1 \mathrm{mg}$ of AVG per 1 and were inoculated with $R$. leguminosarum bv. viciae strain 248, were grown for 2 days at $28^{\circ} \mathrm{C}$ on YMB medium, $\mathrm{OD}_{660}=10^{-1}$, diluted 1:100 to 2 $\times 10^{6}$ bacteria per $\mathrm{ml}$. Then, $10^{-4} \mathrm{M} \mathrm{SA}$ was added, and the plants were allowed to grow for 12 days in a growth chamber similar to that used for $V$. sativa.

Seeds of $T$. repens $\mathrm{L}$. were treated in the same way as $P$. sativum seeds, except that the sulfuric acid treatment was for $5 \mathrm{~min}$, and the bleach treatment was for $10 \mathrm{~min}$. Germination

Table 1. Bacterial strains and their relevant characteristics

\begin{tabular}{|c|c|c|}
\hline Strains & Relevant characteristics $^{\mathrm{a}}$ & References \\
\hline \multicolumn{3}{|l|}{ Rhizobium } \\
\hline 248 & R. leguminosarum bv. viciae wild type containing Sym plasmid pRL1JI & Josey et al. 1979 \\
\hline RBL 5515 & LPR5 R. leguminosarum bv. trifolii cured of its own Sym plasmid $\operatorname{Str}^{\mathrm{r}} \mathrm{Rif}^{\mathrm{r}}$ & Priem and Wijffelman 1984 \\
\hline RBL 5523 & RBL 5515 with Sym plasmid pRL1JI derived from $R$. leguminosarum bv. viciae $\operatorname{Str}^{\mathrm{r}} \operatorname{Rif}^{\mathrm{r}} \mathrm{Spc} 3:: \operatorname{Tn} 1831$ & Pees et al. 1986 \\
\hline LPR 5045 & R. leguminosarum bv. trifolii RCR5 cured of its own Sym plasmid Rif ${ }^{\mathrm{r}}$ & Hooykaas et al. 1982 \\
\hline RBL 5561 & LPR 5045 with pRL1JI nodD2::Tn5 Rif2 ${ }^{\mathrm{r}}$ & Zaat et al. 1987 \\
\hline RBL 5562 & LPR 5045 with pRL1JI nodA10::Tn5 Rif2 ${ }^{\mathrm{r}}$ & Wijffelman et al. 1985 \\
\hline RBL 5602 & LPR 5045 with pRL1JI nodE1::Tn5 $\mathrm{Str}^{\mathrm{r}} \mathrm{Rif}^{\mathrm{r}} \mathrm{Spc}^{\mathrm{r}}$ & Wijffelman et al. 1985 \\
\hline RBL 5797 & LPR 5045 with pRL1JI nodO d4::Tn5 Rif2 ${ }^{\mathrm{r}}$ & Spaink et al. 1992 \\
\hline A 91 & R. leguminosarum bv. viciae nodE68::Tn5 nodO93::Tn3 HoHo1 & Walker and Downie 2000 \\
\hline TONO & Mesorhizobium loti wild type & Banba et al. 2001 \\
\hline E1R & Rif $^{\mathrm{T}}$ derivative of wild type $M$. lot $i$ isolate E1 & López-Lara et al. 1995 \\
\hline $\mathrm{R} 7 \mathrm{~A}$ & Rif $^{T}$ derivative of wild type $M$. loti NZP 2238 & Sullivan et al. 1995 \\
\hline RCR 2011 & Sinorhizobium meliloti wild type & Faucher et al. 1988 \\
\hline ANU 843 & R. leguminosarum bv. trifolii wild type & Rolfe et al. 1980 \\
\hline CE3 & R. etli wild type & Vásquez et al. 1991 \\
\hline HN01 & S. fredii wild type & Chen et al. 1995 \\
\hline \multicolumn{3}{|l|}{ Plasmids } \\
\hline pMP 604 & IncP $\mathrm{Tc}^{\mathrm{r}}$ contains nodD FITA (flavonoid independent transcription activation) & Spaink et al. 1989 \\
\hline pXLGD4 & IncP pGD499 prime carrying a hemA::lac $Z$ fusion & Leong et al. 1985 \\
\hline
\end{tabular}

\footnotetext{
${ }^{\mathrm{a}}$ Inc = plasmid incompatibility group; $\mathrm{Rif}^{\mathrm{r}}, \mathrm{Spc}^{\mathrm{r}}, \mathrm{Str}^{\mathrm{r}}$, and $\mathrm{Tc}^{\mathrm{r}}=$ rifampicin, spectinomycin, streptomycin, and tetracycline resistance; and $\mathrm{Tn}_{1831}$ codes
} for spectinomycin and streptomycin resistance. 
was overnight at $28^{\circ} \mathrm{C}$ on agar plates with Jensen medium. Seeds with roots about $2.5 \mathrm{~cm}$ long were selected and transferred to dark pots containing liquid Jensen medium with $0.025 \mathrm{mg}$ of AVG per 1, were inoculated with $R$. leguminosarum bv. trifolii strain ANU 843, were grown for 2 days at $28^{\circ} \mathrm{C}$ on $\mathrm{YMB}$ medium, $\mathrm{OD}_{660}=10^{-4}$, diluted $1: 1,000$ to 800 bacteria per $\mathrm{ml}$. Then, $10^{-4} \mathrm{M} \mathrm{SA}$ was added, and the plants were allowed to grow for 7 days in the same growth chamber as V. sativa.

Seeds of $M$. sativa were treated in the same way as $T$. repens, except that, before germination, the seeds were incubated for $24 \mathrm{~h}$ at $4^{\circ} \mathrm{C}$. Seeds with roots $2.5 \mathrm{~cm}$ long were selected, were transferred to dark pots containing Jensen medium, MES buffer, pH 5.6, $0.1 \mathrm{mg}$ of AVG per 1, were inoculated with $S$. meliloti strain RCR 2011, were grown for 2 days at $28^{\circ} \mathrm{C}$ on TY medium (Ardourel et al 1994), $\mathrm{OD}_{660}=10^{-3}$, were diluted $1: 1,000$ to $2 \times 10^{3}$ bacteria per $\mathrm{ml}$. Then, $10^{-4} \mathrm{M} \mathrm{SA}$ was added, and the plants were allowed to grow for 11 days in the same growth chamber as V. sativa.

Seeds of $G$. soja were treated in the same way as $T$. repens except, before germination, the seeds were incubated for 7 days at $4^{\circ} \mathrm{C}$. Seeds with roots $2.5 \mathrm{~cm}$ long were selected, were transferred to dark pots containing Jensen medium, were buff-

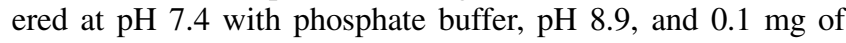
AVG per ml, were inoculated with $S$. fredii strain $\mathrm{HN} 01$, were grown for 2 days at $28^{\circ} \mathrm{C}$ on $\mathrm{YMB}$ medium, $\mathrm{OD}_{660}=10^{-3}$, diluted 1:500 to $10^{4}$ bacteria per $\mathrm{ml}$. Then, $10^{-4} \mathrm{M} \mathrm{SA}$ was added, and the plants were allowed to grow for 8 days in the same growth chamber as for V. sativa.

Seeds of Phaseolus vulgaris cv. Negro Jamapa were grown according to the method described in Van Spronsen and associates (2001). Briefly, after sterilization, incubation for $24 \mathrm{~h}$ at $4^{\circ} \mathrm{C}$, and germination for 2 days at $28^{\circ} \mathrm{C}$, seeds with roots about $2 \mathrm{~cm}$ long were selected and were transferred to brown pots with Fåhraeus medium and $0.1 \mathrm{mg}$ of AVG per ml, were inoculated with $R$. etli strain CE3, were grown two days at $28^{\circ} \mathrm{C}$ on $\mathrm{YMB}$ medium, $\mathrm{OD}_{660}=10^{-1}$, were diluted $1: 250$ to 8 $\times 10^{5}$ bacteria per $\mathrm{ml}$. Then, $10^{-4} \mathrm{M} \mathrm{SA}$ was added, and the plants were allowed to grow for 11 days at $24^{\circ} \mathrm{C}$.

\section{Microscopy.}

Whole roots were cut from the plants and kept in 50 or $70 \%$ ethanol. The roots were examined with an MZ 12 stereomicroscope equipped with a transmitted-light stand for bright and dark field viewing (Leica Microsystems, Wetzlar, Germany). Images were recorded with a DKC-5000 digital photo camera (Sony, Tokyo), were converted to grayscale, and were corrected for brightness and contrast using Adobe Photoshop software (San Jose, CA, U.S.A.). $\beta$-Galactosidase activity in and on $V$. sativa and L. japonicus roots was assayed as described previously (Boivin et al 1990).

\section{SA determination.}

Extraction and quantification of SA was done according to Verberne and associates (2000). Free SA was quantified by chromatography on a Phenomenex column (Metrohm, Herisau, Switzerland), type LUNA $3 \mu \mathrm{C} 18,150 \times 4.6 \mathrm{~mm} 3 \mu \mathrm{m}$. The eluent of $90 \% 0.2 \mathrm{M}$ sodium acetate, $\mathrm{pH} 5.5$, and $10 \%$ methanol had a flow rate of $0.8 \mathrm{ml}$ per min. SA was detected with a Shimadzu (Kyoto, Japan) RF-10Axl spectrofluorometric detector, using an emission wavelength of $407 \mathrm{~nm}$ and excitation wavelength of $305 \mathrm{~nm}$.

\section{ACKNOWLEDGMENTS}

The authors wish to thank P. Hock for preparing the line drawings. We also thank the following people for providing bacteria: M. Kawaguchi,
University of Tokyo for M. loti strain TONO, M. Lihong, Huazhong Agricultural University, Wuhan, China, for S. fredii strain HN01, and J. A. Downie, John Innes Centre, the Biotechnology and Biological Sciences Research Council (BBSRC), Norwich, U.K., for R. leguminosarum bv. viciae strain A91. The supernodulating pea P. sativum L. cv. Frisson P88 was a kind gift from M. Sagan, Station de Génétique et d'Amélioration des Plantes, Dijon, France. K. J. M. B. was supported by a NWO-STW grant (project number LBI 4572).

\section{LITERATURE CITED}

Ardourel, M., Demont, N., Debellé, F., Maillet, F., de Billy, F., Promé, J. C., Dénarié, J., and Truchet, G. 1994. Rhizobium meliloti lipooligosaccharide nodulation factors: Different structural requirements for bacterial entry into target root hair cells and induction of plant symbiotic developmental responses. Plant Cell 6:1357-1374.

Baier, R., Schiene, K., Kohring, B., Flaschel, E., and Niehaus, K. 1999. Alfalfa and tobacco cells react differently to chitin oligosaccharides and Sinorhizobium meliloti nodulation factors. Planta 210:157-164.

Banba, M., Siddique, A.-B. M., Kouchi, H., Izui, K., and Hata, S. 2001. Lotus japonicus forms early senescent root nodules with Rhizobium etli. Mol. Plant-Microbe Interact. 14:173-180

Blilou, I., Ocampo, J. A., and García-Garrido, J. M. 1999. Resistance of pea roots to endomycorrhizal fungus or Rhizobium correlates with enhanced levels of endogenous salicylic acid. J. Exp. Bot. 50:1663-1668.

Boivin, C., Camut, S., Malpica, C. A., Truchet, G., and Rosenberg, C. 1990. Rhizobium meliloti genes encoding catabolism of trigonelline are induced under symbiotic conditions. Plant Cell 2:1157-1170.

Bol, J. F., Buchel, A. S., Knoester, M., Baladin, T., van Loon, L. C., and Linthorst, H. J. M. 1996. Regulation of the expression of plant defence genes. Plant Growth Reg. 18:87-91.

Bueno, P., Soto, M. J., Rodríguez-Rosales, M. P., Sanjuan, J., Olivares, J., and Donaire, J. P. 2001. Time-course of lipoxygenase, antioxidant enzyme activities and $\mathrm{H}_{2} \mathrm{O}_{2}$ accumulation during the early stages of Rhizobium-legume symbiosis. New Phytol. 152:91-96.

Chen, W. L., Zhou, J. C., Hung, Q. Y., and Li, F. D. 1995. Diversity of Sym plasmid in Rhizobium fredii strain and plasmid stability under free-living and symbiotic condition. Pages 116-125 in: Diversity and Taxonomy of Rhizobia. F. D. Li, ed., China Agricultural Scientech Press, Beijing, China.

Conrath, U., Chen, Z., Ricigliano, J. R., and Klessig, D. F. 1995. Two inducers of plant defense responses, 2,6-dichloroisonicotinic acid and salicylic acid, inhibit catalase activity in tobacco. Proc. Natl. Acad. Sci. U.S.A. 92:7143-7147.

Faucher, C., Maillet, F., Vasse, J., Rosenberg, C., van Brussel, A. A. N., Truchet, G., and Dénarié, J. 1988. Rhizobium meliloti host range nodH determines production of an alfalfa-specific extracellular signal. J. Bacteriol. 170:5489-5499.

Hooykaas, P. J. J., Klapwijk, P. M., Nuti, M. P., Schilperoort, R. A., and Rörsch, A. 1977. Transfer of the Agrobacterium tumefaciens Ti plasmid to avirulent agrobacteria and to rhizobia ex planta. J. Gen. Microbiol. 98:477-484.

Hooykaas, P. J. J., Schnijdewindt, F. G. M., and Schilperoort, R. A. 1982. Identification of the Sym plasmid of Rhizobium leguminosarum strain 1001 and its transfer to and expression in other Rhizobia and Agrobacterium tumefaciens. Plasmid 8:73-82.

Josey, D. P., Beynon, J. L., Johnston, A. W. B., and Beringer, J. E. 1979. Strain identification in Rhizobium using intrinsic antibiotic resistance. J. Appl. Microbiol. 46:343-350.

Kamst, E., Spaink, H. P., and Kafetzopoulos, D. 1998. Biosynthesis and secretion of rhizobial lipochitin-oligosaccharide signal molecules. Pages 29-71 in: Subcellular Biochemistry. Vol. 29. Plant Microbe Interactions. B. B. Biswas and H. K Das, eds., Plenum Press, New York.

Leong, S. A., Williams, P. H., and Ditta, G. S. 1985. Analysis of the 5' regulatory region of the gene for $\delta$-aminolevulinic acid synthase of Rhizobium meliloti. Nucleic Acids Res. 13:5965-5976

Levitan, H., Barker, J. L. 1972. Salicylate: A structure-activity study of its effects on membrane permeability. Science 176:1423-1425.

López-Lara, I. M., van den Berg, J. D. J., Thomas-Oates, J. E., Glushka, J., Lugtenberg, B. J. J., and Spaink, H. P. 1995. Structural identification of the lipo-chitin oligosaccharide nodulation signals of Rhizobium loti. Mol. Microbiol. 15:627-638.

Malamy, J., Carr, J. P., Klessig, D. F., and Raskin, I. 1990. Salicylic acid: A likely endogenous signal in the resistance response of tobacco to viral infection. Science 250:1002-1004.

Marnett, L. J., Rowlinson, S. W., Goodwin, D. C., Kalgutkar, A. S., and Lanzo, C. A. 1999. Arachidonic acid oxygenation by COX-1 and COX-2. J. Biol. Chem. 274:22903-22906.

Martínez-Abarca, F., Herrera-Cervera, J. A., Bueno, P., Sanjuan, J., 
Bisseling, T., and Olivares, J. 1998. Involvement of salicylic acid in the establishment of the Rhizobium meliloti-alfalfa symbiosis. Mol. Plant-Microbe Interact. 11:153-155

Pees, E., Wijffelman, C. A., Mulders, I., van Brussel, A. A. N., and Lugtenberg, B. J. J. 1986. Transposition of Tn1831 to Sym plasmids of Rhizobium leguminosarum and Rhizobium trifolii. FEMS (Fed. Eur. Microbiol. Soc.) Microbiol. Lett. 33:165-171

Peng, H. M., Dreyer, D. A., VandenBosch, K. A., and Cook, D. 1996. Gene structure and differential regulation of the Rhizobium-induced peroxidase gene rip1. Plant Physiol. 112:1437-1446.

Priem, W. J. E., and Wijffelman, C. A. 1984. Selection of strains cured of the Rhizobium leguminosarum Sym plasmid pRL1JI by using small bacteriocin. FEMS (Fed. Eur. Microbiol. Soc.) Microbiol. Lett. 25:247-251.

Ramu, S. K., Peng, H. M., and Cook, D. R. 2002. Nod factor induction of reactive oxygen species production is correlated with expression of the early nodulin gene ripl in Medicago truncatula. Mol. Plant-Microbe Interact. 15:522-528

Röhrig, H., Schmidt, J., Miklashevichs, E., Schell, J., and John, M. 2002. Soybean ENOD40 encodes two peptides that bind to sucrose synthase. Proc. Natl. Acad. Sci. U.S.A. 99:1915-1920.

Rolfe, B. G., Gresshoff, P. M., and Shine, J. 1980. Rapid screening method for symbiotic mutants of Rhizobium leguminosarum biovar trifolii on white clover plants. Plant Sci. Lett. 19:277-284.

Rüffer, M., Steipe, B., and Zenk, M. H. 1995. Evidence against specific binding of salicylic acid to plant catalase. FEBS (Fed. Eur. Biochem. Soc.) Lett. 377:175-180.

Sagan, M., and Gresshoff, P. M. 1996. Developmental mapping of nodulation events in pea (Pisum sativum L.) using supernodulating plant genotypes and bacterial variability reveals both plant and Rhizobium control of nodulation regulation. Plant Sci. 117:167-179.

Santos, R., Hérouart, D., Sigaud, S., Touati, D., and Puppo, A. 2001. Oxidative burst in Alfalfa-Sinorhizobium meliloti symbiotic interaction. Mol. Plant-Microbe Interact. 14:86-89.

Schaller, F. 2001. Enzymes of the biosynthesis of octadecanoid-derived signaling molecules. J. Exp. Bot. 52:11-23.

Spaink, H. P., Wijffelman, C. A., Okker, R. J. H., and Lugtenberg, B. J. J. 1989. Localization of functional regions of the Rhizobium nodD product using hybrid nodD genes. Plant Mol. Biol. 12:59-73.

Spaink, H. P., Sheely, D. M., van Brussel, A. A. N., Glushka, J., York, W. S., Tak, T., Geiger, O., Kennedy, E. P., Reinhold, V. N., and Lugtenberg, B. J. J. 1991. A novel highly unsaturated fatty acid moiety of lipo-oligosaccharide signals determines host specificity of Rhizobium. Nature 354:125-130.
Spaink, H. P., Aarts, A., Stacey, G., Bloemberg, G. V., Lugtenberg, B. J. J., and Kennedy, E. P. 1992. Detection and separation of Rhizobium and Bradyrhizobium Nod metabolites using thin-layer chromatography. Mol. Plant-Microbe Interact. 5:72-80.

Stougaard, J. 2000. Regulators and regulation of legume root nodule development. Plant Physiol. 124:531-540.

Sullivan, J. T., Patrick, H. N., Lowther, W. L., Scott, D. B., and Ronson, C. W. 1995. Nodulation strains of Rhizobium loti arise through chromosomal symbiotic gene transfer in the environment. Proc. Natl. Acad. Sci. U.S.A. 92:8985-8989.

Van Brussel, A. A. N., Tak, T., Boot, K. J. M., and Kijne, J. W. 2002. Autoregulation of root nodule formation: Signals of both symbiotic partners studied in a split-root system of Vicia sativa subsp. nigra. Mol. Plant-Microbe Interact. 15:341-349.

Van Spronsen, P. C., Grønlund, M., Pacios-Bras, C., Spaink, H. P., and Kijne, J. W. 2001. Cell biological changes of outer cortical root cells in early determinate nodulation. Mol. Plant-Microbe Interact. 14:839-847.

Van Workum, W. A. T., van Slageren, S., van Brussel, A. A. N., and Kijne, J. W. 1998. Role of exopolysaccharides of Rhizobium leguminosarum bv. viciae as host plant-specific molecules required for infection thread formation during nodulation of Vicia sativa. Mol. PlantMicrobe Interact. 11:1233-1241

Vásquez, M., Dávalos, A., de las Peňas, A., Sánchez, F., and Quinto, C. 1991. Novel organization of the common nodulation genes in Rhizobium leguminosarum bv. phaseoli strains. J. Bacteriol. 173:1250-1258

Verberne, M. C., Verpoorte, R., Bol, J. F., Mercado-Blanco, J., and Linthorst, H. J. M. 2000. Overproduction of salicylic acid in plants by bacterial transgenes enhances pathogen resistance. Nat. Biotechnol. 18:779-783.

Vincent, J. M. 1970. A manual for the practical study of root-nodule bacteria. I.B.P. Handbook No. 15. Blackwell Scientific Publications, Oxford, U.K

Walker, S. A. and Downie, J. A. 2000. Entry of Rhizobium leguminosarum bv. viciae into root hairs requires minimal Nod Factor specificity, but subsequent infection thread growth requires nodO or nodE. Mol. Plant-Microbe Interact. 13:754-762

Wijffelman, C. A., Pees, E., van Brussel, A. A. N., Okker, R. J. H., and Lugtenberg, B. J. J. 1985. Genetic and functional analysis of the nodulation region of the Rhizobium leguminosarum Sym plasmid pRL1JI. Arch. Microbiol. 143:225-232.

Zaat, S. A. J., Wijffelman, C. A., Spaink, H. P., van Brussel, A. A. N., Okker, R. J. H., and Lugtenberg, B. J. J. 1987. Induction of the nodA promotor of Rhizobium leguminosarum sym plasmid pRL1JI by plant flavanones and flavones. J. Bacteriol. 169:198-204. 